Highly Energetic Physical Processes and

Mechanisms for Emission from Astrophysical Plasmas

IAU Symposium, Vol. 195, 2000

P. C. H. Martens, S. Tsuruta, and M. A. Weber, eds.

\title{
X-Ray Emission as an Indicator of Cosmic Ray Acceleration in Supernova Remnants
}

\author{
R. Petre \\ Laboratory for High Energy Astrophysics, Goddard Space Flight Center, \\ Greenbelt, MD 20771, U.S.A. \\ G. E. Allen
}

NASA / Chandra X-Ray Center, Massachusetts Institute of Technology, Cambridge MA, 02139, U.S.A.

U. Hwang

Goddard Space Flight Center and University of Maryland, Greenbelt, MD 20771, U.S.A.

J. W. Keohane

NASA / North Carolina School of Science and Mathematics, Durham, NC 27705, U.S.A.

E. V. Gotthelf

Astronomy Department, Columbia University, New York, NY 10027, U.S.A.

\begin{abstract}
X-ray observations over the past several years have led to the discovery of nonthermal X-ray emission arising in the shells of many young supernova remnants, including SN 1006, Cas A, and Tycho. This emission is thought to be synchrotron emission from electrons that have been shock accelerated to hundreds of $\mathrm{TeV}$, and thus represents strong evidence that cosmic rays are accelerated in SNR shocks. The X-ray observations are corroborated by detection of $\mathrm{TeV}$ gamma rays from two of these remnants. A systematic investigation of young, shell-like remnants suggests that the nonthermal X-ray emission from shock-accelerated electrons is a common, if not ubiquitous, feature. We review the status of the X-ray observations and describe how they can be used to provide insight into the shock acceleration process.
\end{abstract}

\section{Introduction}

It has been postulated for many years that cosmic rays with energy as high as the $\sim 3,000 \mathrm{TeV}$ spectral turnover (or "knee") are produced by diffusive shock acceleration in Galactic supernova remnants (SNRs). The acceleration sites cannot be observed directly because the intervening Galactic magnetic fields curve the trajectories of these energetic particles. Detection of synchrotron 
emission from SNR shells in the radio band verify that electrons with energies up to the $\mathrm{GeV}$ range are accelerated there. Detections of $\gamma$-rays from a few evolved SNRs has been interpreted to indicate that these shocks also produce $\mathrm{GeV}$ protons (c.f., Esposito et al. 1996). Until recently, however, it has not been possible to search for evidence of particles with energy closer to the knee. In the IR, visible, UV, and X-ray bands, the surface brightness of the synchrotron spectrum extrapolated from the radio is substantially lower than that of thermal emission from shock-heated gas. Only above $\sim 5 \mathrm{keV}$, where the thermal emission peters out, might it be possible to detect synchrotron emission in the form of a hard continuum component, but only if electrons are accelerated to sufficiently high energy, on the order of $100 \mathrm{TeV}$.

The broad bandpass, high sensitivity, and moderate resolution spectral capabilities of the latest generation of X-ray observatories, most notably ASCA and RXTE, have led to a breakthrough in our ability to detect evidence for highly relativistic particles in SNR shells. ASCA observations of the supernova remnant SN 1006 revealed that its low surface brightness regions have the thermal spectrum characteristic of reverse-shocked ejecta, but that the radio and X-ray bright northeastern and southwestern limbs have featureless X-ray spectra that can be modeled as power laws with (energy) spectral index $\alpha \sim 2$. The most plausible explanation of this emission is synchrotron radiation from electrons with energies up to $\sim 200 \mathrm{TeV}$. As acceleration processes at these highly relativistic energies make no distinction between positively and negatively charged particles, we infer that protons and nuclei are being accelerated to these energies as well, and therefore SN 1006 is the first identified cosmic ray source (Koyama et al. 1995). Theoretical models support this conclusion (c.f., Reynolds 1996) or show that alternative interpretations fail (c.f., Laming 1998). This conclusion has been dramatically confirmed by the subsequent detection of $\mathrm{TeV} \gamma$-rays from SN 1006, which arise from Compton scattering of cosmic microwave background photons off the TeV electrons (Tanimori et al. 1998).

\section{Current Results}

SN 1006 is an unusual (but apparently not unique) SNR in that the normally dominant thermal X-ray flux from the shock front is relatively low, allowing the synchrotron $\mathrm{X}$-rays from the accelerated electrons to be easily detectable even at $1 \mathrm{keV}$. This is not the case for other young SNRs with high enough shock velocity to accelerate particles to $\mathrm{TeV}$ energies, most likely because of their expansion into a higher density medium. Thus, for most remnants, the search for evidence of cosmic ray acceleration is considerably more difficult. Nevertheless, hard components have been found in the ASCA and RXTE spectra of several other SNRs. Table 1 summarizes the results to date. The strongest cases for this tail being due to shock-accelerated TeV electrons are Cas A (Allen et al. 1997) and G347.5-0.5 (= RX J1713.7-3946; Koyama et al. 1996). In Figure 1, we show the RXTE spectra of five remnants with nonthermal components.

The remnants in Table 1 share two important attributes. i.) Each has a continuum X-ray component extending to energies beyond the thermal emission. If only the $\mathrm{X}$-ray spectrum is considered, this tail can be characterized as a power law with $\alpha \sim 2$ (in contrast to $\alpha \sim 1$ for plerions). A broader view of the 
Table 1. Galactic SNR's with Hard, Nonthermal X-Ray Spectral Components.

\begin{tabular}{l|cccl}
\hline Object & $\begin{array}{c}\text { Age } \\
(\mathrm{ky})\end{array}$ & $\begin{array}{c}\mathrm{R} / \mathrm{X} \\
\text { Match? }\end{array}$ & Type & Result \\
\hline Cas A & 0.3 & Y & II & Synchrotron tail extends to $\geq 100 \mathrm{keV}$ \\
Kepler & 0.4 & Y & II? & $\alpha \sim 2.5$ \\
Tycho & 0.4 & N & Ia & Tail with $\alpha \sim 2$ extends $\geq 20 \mathrm{keV}$ \\
G266.3-1.2 & $\sim 1$ & $?$ & II? & RXTE shows $\alpha \sim 2 ;$ low $n$ medium \\
SN1006 & 1.0 & Y & Ia & Obvious nonthermal shell; TeV $\gamma$-rays \\
G347.5-0.5 & $\sim 1$ & $?$ & II? & Nonthermal spectrum; $\alpha \sim 1.5 ;$ low $n$ \\
W49B & $1-3$ & Y & $?$ & Hard X-ray continuum has $\alpha \sim \alpha$ 1006 \\
RCW 86 & $\sim 2$ & N & II? & Tail has $\alpha \sim 2$ extending $\geq 20$ keV \\
IC 443 & $1-4$ & N & II & $\begin{array}{l}\text { NT flux from enhanced acceleration } \\
\text { due to strong shock/cloud collision }\end{array}$ \\
W50 & & & & NT flux from electrons accelerated in \\
\end{tabular}

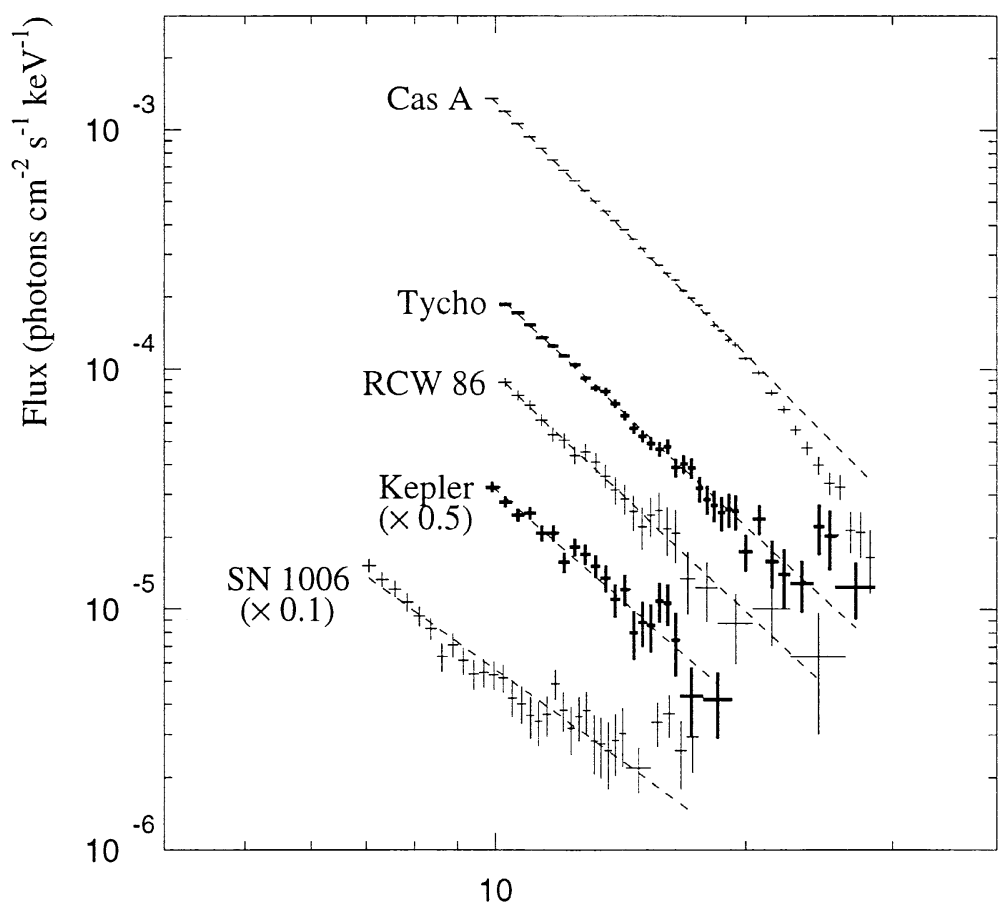

Photon Energy (keV)

Figure 1. RXTE PCA spectra of five remnants with hard nonthermal tails probably produced by synchrotron emission from $\mathrm{TeV}$ electrons. Each spectrum is characterized by a power law with spectral index $\sim 2$. 
spectrum requires that this tail be a continuation of the radio synchrotron spectrum, with a rollover at, typically, $100 \mathrm{eV}$ (Reynolds \& Keohane 1999). ii.) For those remnants in which we can isolate spectrally the nonthermal component, we find a morphological match between the radio emission and the nonthermal $\mathrm{X}$-rays. This is, of course, expected if the same mechanism produces the X-ray and radio emission.

Meticulous analysis was required to establish the presence of a nonthermal component in some of these remnants. In Tycho's SNR, for instance, there is no evidence for this component in either the ASCA spectrum or hard band Xray image; only by simultaneous fitting of the ASCA and RXTE spectra does the evidence become strong. Kepler represents an even subtler case: only after extremely careful spectral modeling does the need for a nonthermal component become apparent (Decourchelle \& Petre 1999). Also, the presence of a hard continuum component is not in itself sufficient to demonstrate the presence of $\mathrm{TeV}$ particles. Hard components could be produced by any of a number of mechanisms: a hot thermal component, possibly associated with the forward shock (for most SNRs, ASCA lacks the bandwidth to discriminate between a thermal and nonthermal model), nonthermal bremsstrahlung (Asvarov et al. 1990), bremsstrahlung from ions accelerated to MeV energy (Tatischeff, Ramaty, \& Kozlovsky 1998), and even synchrotron emission from a hidden pulsar. The data from every SNR must be carefully scrutinized to determine whether the hard component truly arises from TeV electrons.

Two remnants in Table 1 are especially notable: G347.5-0.5 (= RX J1713.73913) and G266.3-1.2 (= RX J0852.0-4622). G347.5-0.5 has been described as the "twin" of SN 1006. Indeed, it has many similar properties, including a synchrotron-dominated spectrum throughout the X-ray band and a very low radio surface brightness. Most importantly, recent observations detect $\mathrm{TeV} \gamma$ radiation (Muraishi et al. 1999), making it the second remnant after SN1006 for which the presence of $\mathrm{TeV}$ electrons has been confirmed. G266.3-1.2 is probably the closest young SNR (Aschenbach 1998; Iyudin et al. 1998). Like SN 1006 and G347.5-0.5, it is expanding into a very low density medium. Our RXTE observations show no evidence of $\mathrm{Fe}$ line emission (trivially detected in other shell-like historical remnants) and a continuum spectrum consistent with a power law of $\alpha \sim 2$. This is likely the closest high-energy cosmic ray source.

\section{Discussion}

The research sparked by the realization that the X-rays from SN 1006 provide evidence for cosmic ray acceleration has led to several findings:

1. As indicated in Table 1, all known young, shell-like Galactic SNRs have hard nonthermal tails indicative of cosmic ray acceleration. Thus, cosmic ray acceleration is probably ubiquitous in young Galactic SNRs.

2. There might be more than one mechanism for producing $\mathrm{TeV}$ electrons, and thus possibly cosmic rays, in SNRs. In the middle-aged remnant IC 443, for instance, hard emission is localized to regions along the eastern rim where the SNR shock is most strongly interacting with a molecular 


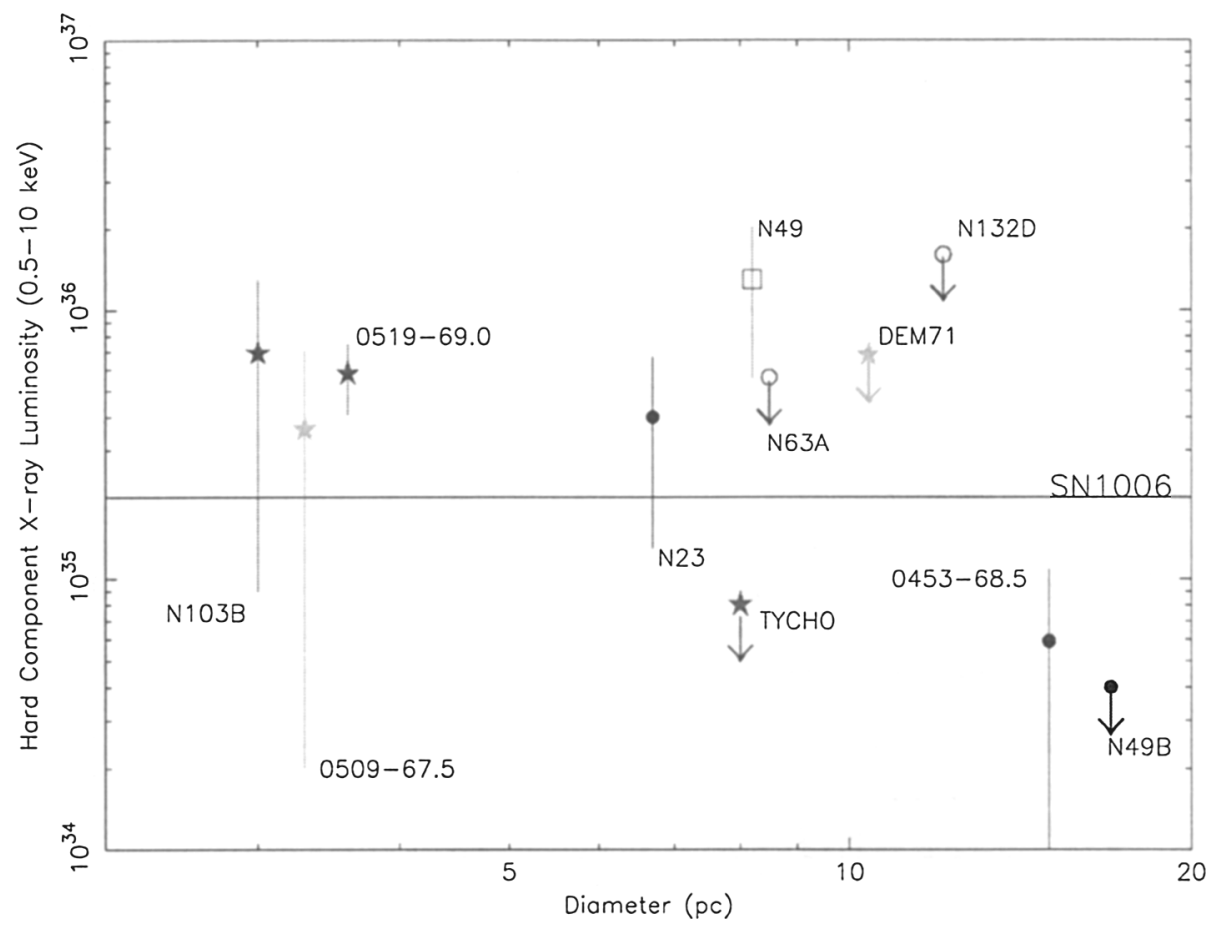

Figure 2. Luminosity in $0.5-10.0 \mathrm{keV}$ band of hard components versus diameter for a sample of LMC SNRs. Symbols indicate progenitor type (stars for Type Ia, circles for core-collapse, open square for N49 because of presence of compact SGR). For the younger (smaller diameter) remnants, many could easily "hide" a nonthermal component with the luminosity of that of SN 1006, though for many of these remnants at least some of the hard flux is thermal. The maximum hard component flux from the two largest and presumably oldest remnants is substantially less than that from the others. This suggests a reduction or termination of production of $\mathrm{TeV}$ electrons.

cloud (Keohane et al. 1997). Hydrodynamical modeling indicates that enhanced particle acceleration can occur downstream from shock interactions with isolated cloudlets (Jones \& Kang 1993), and this has been proposed as the mechanism to explain the hard emission in IC 443. The hard, nonthermal X-ray emission in the lobes of the old remnant W50 are thought to be produced by particle acceleration at the terminal shocks of the jets emanating from SS 433 (Safi-Harb \& Petre 1999). 
Particle Rollover Energy vs. Time in SNR Shocks

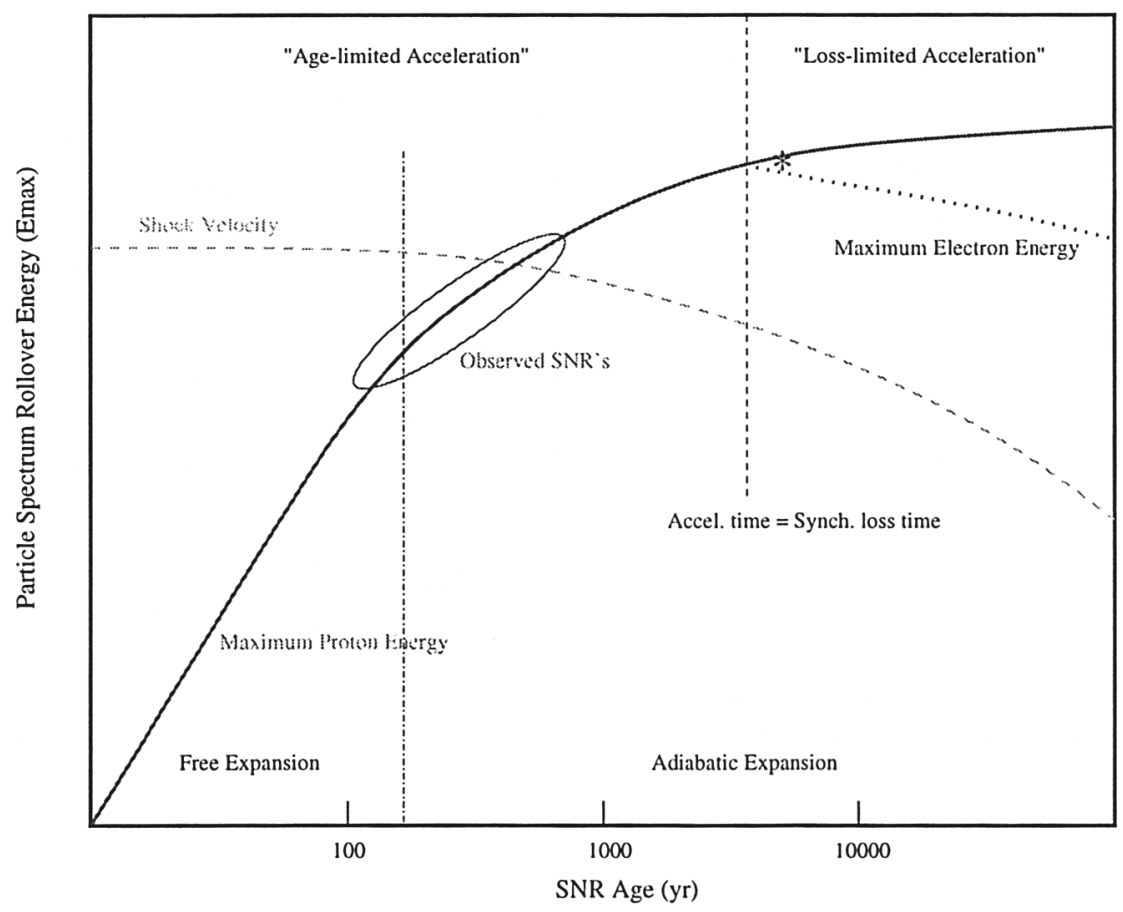

Figure 3. Schematic evolution of turnover energy for electrons and protons in an SNR shock. Electron and proton maximum energy stays coupled well into the adiabatic phase, when synchrotron losses begin to affect the electron population. None of the SNRs with detected synchrotron tails have reached this decoupling time, so the electron spectrum should reflect the proton spectrum. (This figure modelled after Reynolds \& Keohane 1999.)

3. The X-ray luminosity of the hard component evolves with time. As summarized in Figure 2, a systematic study of the ASCA spectra of bright LMC SNRs indicates that the larger and presumably older remnants in the sample show no evidence for hard, nonthermal emission, while the smaller, younger remnants easily can have a nonthermal luminosity comparable with that of SN 1006 (Petre et al. 1997). This provides evidence that the $\mathrm{TeV}$ electron population evolves.

4. A new class of SNRs is emerging with SN 1006 as the prototype. These remnants are shell-like, have relatively low radio and X-ray surface bright- 
ness, and their $\mathrm{X}$-rays are primarily produced by synchrotron emission from $\mathrm{TeV}$ electrons. Members of this class include G347.5-0.5 and G266.31.2. These remnants are evolving in a low-density medium in which insufficient material is located to produce a strong reverse shock. Thus, the $\mathrm{X}$-ray emission from the reverse-shocked ejecta, the brightest component in most young remnants, is weak or absent. This class contains at least one Type Ia remnant (SN 1006); the others could be the remnants of corecollapse explosions. Finding new members of this class is an observational challenge as these remnants' low radio surface brightness causes them to be overlooked or not detected in surveys. At least one other candidate exists, however: G182.4+4.3 is a low radio surface brightness remnant near the Galactic anticenter, with a very low X-ray flux (Kothes, Furst, \& Reich 1998).

5. Finally, despite the clear evidence for cosmic ray acceleration, there remain discrepancies between the observations and theory. One can construct an argument invoking equipartition of energy among the hot gas, magnetic field, and relativistic particle components to argue the plausibility that all cosmic rays up to the knee are produced in SNRs. But the number density of high-energy electrons responsible for the observed synchrotron $\mathrm{X}$-rays is at least an order of magnitude less than what one would expect if SNRs are responsible for all Galactic cosmic rays, provided a comparable number of electrons and protons are accelerated. If a single electron population produces the X-ray and radio synchrotron spectra, then in most SNRs the electron spectrum turns over at energies $\leq 100 \mathrm{TeV}$. Reynolds \& Keohane (1999) argue that the turnover in the observed SNRs is limited by the age; thus, the electrons have not attained the maximum possible energy. However, as the turnover energy is most strongly dependent on the shock velocity, it increases slowly once a remnant enters the adiabatic expansion stage. Moreover, the decoupling between electron and proton acceleration due to the onset of synchrotron losses has not yet occurred; thus, the electron population traces the proton population. This is depicted schematically in Figure 3. This interpretation suggests a potential problem: either acceleration evolves differently from current models or SNRs are not, in fact, responsible for cosmic rays up to the knee.

\section{Conclusion}

In revealing the source of at least some cosmic rays, X-ray astronomy demonstrates once again its capability of addressing fundamental physical issues. This discovery could not have been made without the current generation of X-ray observatories (particularly ASCA and RXTE) combining broad spectral coverage with high sensitivity. The next set of X-ray observatories (Chandra, XMM, and ASTRO-E) will allow us both to search more sensitively for evidence of cosmic ray acceleration in SNRs where it has not yet been found and, via detailed study of the known cosmic ray sources, to probe the physics of the acceleration process. 


\section{References}

Allen, G. E., et al. 1997, ApJ, 487, L97

Aschenbach, B. 1998, Nature, 396, 141

Asvarov, A. I., Dogiel, V. A., Gusienov, O. H., \& Kasumov, F. K. 1990, A\&A, 229,196

Decourchelle, A., \& Petre, R. 1999, Astron. Nachr., 320, 203

Esposito, J. A., Hunter, S. D., Kanbach, G., \& Sreekumar, P. 1996, ApJ, 461, 820

Iyudin, A. F., et al. 1998, Nature, 396, 142

Jones, T. W., \& Kang, H. 1993, ApJ, 402, 560

Keohane, J. W., Petre, R., Gotthelf, E. V., Ozaki, M., \& Koyama, K. 1997, ApJ, 484, 350

Kothes, R., Furst, E., \& Reich, W. 1998, A\&A, 331, 661

Koyama, K., Petre, R., Gotthelf, E. V., Hwang, U., Matsuura, M., Ozaki, M., \& Holt, S. S. 1995, Nature, 378, 255

Koyama, K., et al. 1997, PASJ, 49, L7

Laming, M. 1998, ApJ, 499, L309

Muraishi, H., et al. 1999, astro-ph/9906077, preprint

Petre, R., Hwang, U., Keohane, J. W., \& Gotthelf, E. V. 1997, BAAS, 29, 1267

Reynolds, S. P. 1996, ApJ, 459, L13

Reynolds, S. P., \& Keohane, J. W. 1999, ApJ, 525, 368

Tanimori, T., et al. 1998, ApJ, 497, L25

Tatischeff, V., Ramaty, R., \& Kozlovsky, B. 1998, ApJ, 504, 874

Safi-Harb, S., \& Petre, R. 1999, ApJ, 512, 784 